\title{
Temporal dynamics of the plasma microbiome in recipients at early post-liver transplantation: a retrospective study
}

Toshihiko Okumura ${ }^{1}$, Kazuhiro Horiba ${ }^{1,2,3}$, Hideya Kamei ${ }^{4}$, Suguru Takeuchi ${ }^{1}$, Takako Suzuki ${ }^{1}$, Yuka Torii ${ }^{1}$, Jun-ichi Kawada ${ }^{1}$, Yoshiyuki Takahashi ${ }^{1}$, Yasuhiro Ogura ${ }^{4}$, Tomoo Ogi ${ }^{2,3}$ and Yoshinori Ito ${ }^{1 *}$ (i)

\begin{abstract}
Background: Immunosuppression during liver transplantation (LT) enables the prevention and treatment of organ rejection but poses a risk for severe infectious diseases. Immune modulation and antimicrobials affect the plasma microbiome. Thus, determining the impact of immunosuppression on the microbiome may be important to understand immunocompetence, elucidate the source of infection, and predict the risk of infection in LT recipients. We characterized the plasma microbiome of $\mathrm{LT}$ recipients at early post-LT and assessed the association between the microbiome and clinical events.

Results: In this study, 51 patients who received LT at Nagoya University Hospital from 2016 to 2018 were enrolled. Plasma samples were retrospectively collected at the following time points: 1) within a week after LT; 2) $4 \pm 1$ weeks after LT; 3) $8 \pm 1$ weeks after LT; and 4) within 2 days after a positive blood culture. A total of 111 plasma samples were analyzed using shotgun next-generation sequencing (NGS) with the PATHDET pipeline. Relative abundance of Anelloviridae, Nocardiaceae, Microbacteriaceae, and Enterobacteriaceae significantly changed during the postoperative period. Microbiome diversity was higher within a week after LT than that at 8 weeks after LT. Antimicrobials were significantly associated with the microbiome of LT recipients. In addition, the proportion of Enterobacteriaceae was significantly increased and the plasma microbiome diversity was significantly lower in patients with acute cellular rejection (ACR) than non-ACR patients. Sequencing reads of bacteria isolated from blood cultures were predominantly identified by NGS in 8 of 16 samples, and human herpesvirus 6 was detected as a causative pathogen in one recipient with severe clinical condition.
\end{abstract}

Conclusions: The metagenomic NGS technique has great potential in revealing the plasma microbiome and is useful as a comprehensive diagnostic procedure in clinical settings. Temporal dynamics of specific microorganisms may be used as indirect markers for the determination of immunocompetence and ACR in LT recipients.

Keywords: Acute cellular rejection, Enterobacteriaceae, Liver transplantation, Next-generation sequencing, Plasma microbiome

\footnotetext{
*Correspondence: yoshi-i@med.nagoya-u.ac.jp

'Department of Pediatrics, Nagoya University Graduate School of Medicine,

65 Tsurumai-cho, Showa-ku, Nagoya 466-8550, Japan

Full list of author information is available at the end of the article
}

(c) The Author(s). 2021 Open Access This article is licensed under a Creative Commons Attribution 4.0 International License, which permits use, sharing, adaptation, distribution and reproduction in any medium or format, as long as you give appropriate credit to the original author(s) and the source, provide a link to the Creative Commons licence, and indicate if changes were made. The images or other third party material in this article are included in the article's Creative Commons licence, unless indicated otherwise in a credit line to the material. If material is not included in the article's Creative Commons licence and your intended use is not permitted by statutory regulation or exceeds the permitted use, you will need to obtain permission directly from the copyright holder. To view a copy of this licence, visit http://creativecommons.org/licenses/by/4.0/. The Creative Commons Public Domain Dedication waiver (http://creativecommons.org/publicdomain/zero/1.0/) applies to the data made available in this article, unless otherwise stated in a credit line to the data. 


\section{Background}

Liver transplantation (LT) is a fundamental treatment for patients with end-stage liver diseases. Immunosuppressive medications after LT enable the prevention and treatment of organ rejection but pose a risk for contraction of severe infectious diseases. Therefore, antimicrobials are often required for patients after LT. Immune modulation and antiviral therapies after organ transplantation are known to affect the gut microbiome [1]. In addition, the viral composition of the plasma microbiome after organ transplantation was reported to be affected by antivirals and immunosuppression [2]. Recently, the assessment of association between the microbiome and clinical events is becoming a major research area. However, few studies have evaluated the plasma microbiome, which comprises bacteria, viruses, and fungi, after transplantation.

Next-generation sequencing (NGS) can comprehensively analyze the sequence data of nucleic acids within a sample. Shotgun metagenomic sequencing allows us to obtain all genes in all organisms present in a clinical sample. In the field of clinical microbiology, NGS has enabled us to detect causative pathogens in various infectious diseases [3-6]. The association of the gut microbiome and its effect on pathophysiology of a number of diseases, for example, cardiac failure, cancer, and liver diseases, have been reported [7-10]. In addition to the gut microbiome, multiple microorganism genomes have been detected in blood, which is essentially sterile [11, 12]. Here, we focused on the plasma microbiome and anticipated that it would be useful to predict clinical adverse events after LT, such as acute cellular rejection (ACR) and infectious diseases. Therefore, in this study, we characterized the plasma microbiome of LT recipients. Temporal dynamics of the plasma microbiome in patients who experienced ACR were also investigated. Moreover, shotgun metagenomic sequencing was evaluated as a detection tool of causative pathogens.

\section{Results}

Temporal dynamics of the plasma microbiome

A total of 111 plasma samples were consecutively collected from 51 LT recipients. First, we analyzed 25 recipients whose samples had been collected at all three time points. Subsequently, 28 samples from other patients were analyzed to confirm the results. We obtained an average of 13.5 million reads per sample. Approximately $0.03 \%$ of the total reads were identified as microorganism reads after subtraction of human-derived reads and artifacts (Additional file 1).

Figure 1 shows the time-dependent relative composition of plasma microorganisms. Data include all samples

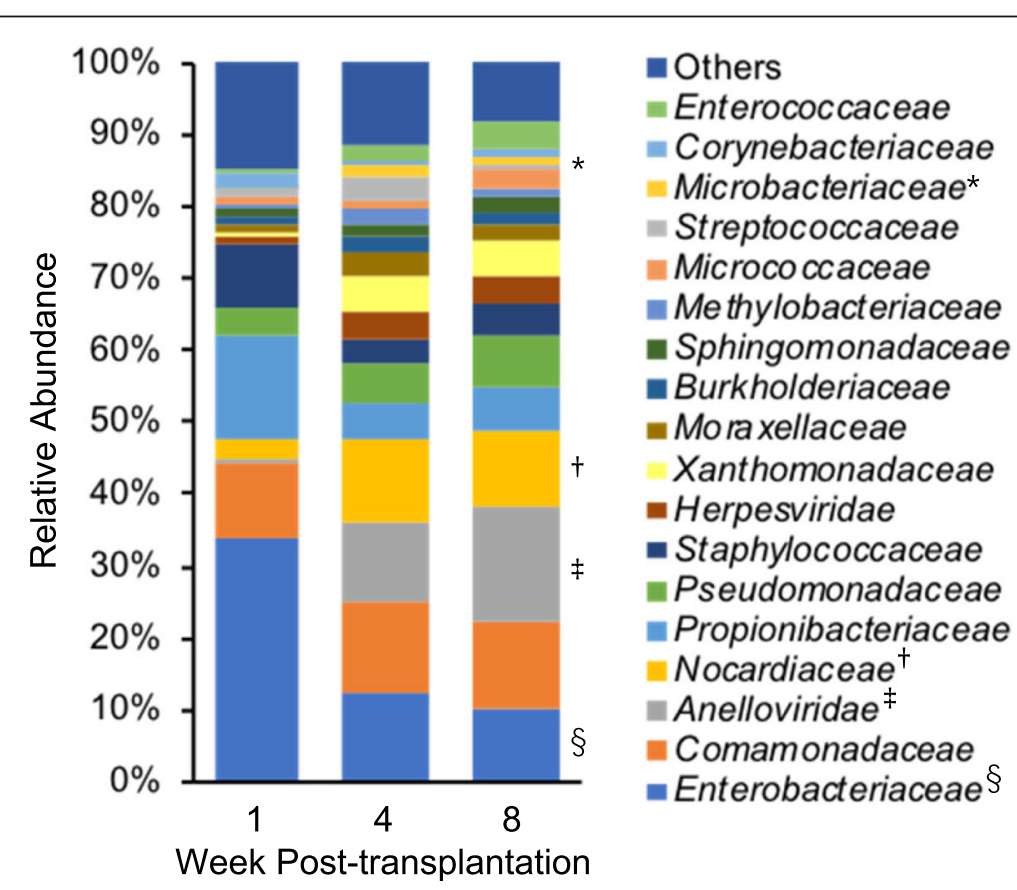

Fig. 1 Change in the relative abundance of each microorganism at the family level in plasma samples after liver transplantation. Microbacteriaceae $(*)$, Nocardiaceae $(\dagger)$, and Anelloviridae $(\ddagger)$ abundance increased in samples collected at week 4 or 8 post-transplantation in comparison with samples collected at week 1 post-transplantation (Anelloviridae: week 4 vs. week 1, $p=0.62$, week 8 vs. week 1, $p<0.001$; Nocardiaceae: week 4 vs. week 1, $p=0.003$, week 8 vs. week 1, $p=0.36$; Microbacteriaceae: week 4 vs. week 1, $p<0.001$, week 8 vs. week 1, $p=$ 0.002). Enterobacteriaceae (§) abundance decreased in samples collected at week 4 or 8 post-transplantation in comparison with samples collected at week 1 post-transplantation (week 4 vs. week $1, p<0.001$, week 8 vs. week $1, p<0.001$ ) 
(46 samples collected at week 1, 28 samples collected at week 4 , and 29 samples collected at week 8 posttransplantation) and show average relative abundance at the family level of taxonomic hierarchy. Anelloviridae, Nocardiaceae, and Microbacteriaceae were significantly increased in samples collected at week 4 or 8 posttransplantation compared with those collected at week 1 post-transplantation (Anelloviridae: $0.07 \pm 0.19$ (week 4) vs. $0.01 \pm 0.04$ (week 1 ), $p=0.62,0.23 \pm 0.28$ (week 8) vs. $0.01 \pm 0.04$ (week 1), $p<0.001$; Nocardiaceae: $0.127 \pm$ 0.100 (week 4) vs. $0.057 \pm 0.084$ (week 1), $p=0.003$, $0.089 \pm 0.074$ (week 8) vs. $0.057 \pm 0.084$ (week 1 ), $p=0.36$; Microbacteriaceae: $0.017 \pm 0.013$ (week 4) vs. $0.004 \pm$ 0.007 (week 1), $p<0.001,0.013 \pm 0.012$ (week 8) vs. $0.004 \pm 0.007$ (week 1), $p=0.002$ ) whereas Enterobacteriaceae was decreased $(0.13 \pm 0.12$ (week 4) vs. $0.26 \pm 0.17$ (week 1), $p<0.001,0.09 \pm 0.11$ (week 8) vs. $0.26 \pm 0.17$ (week 1), $p<0.001$ ). The time-dependent relative compositions of plasma microorganisms at the genus and species levels are shown in Figs. S1 and S2. Figure 2 shows the temporal changes in plasma microbiome diversity. Simpson's diversity index at the genus level at week 1 posttransplantation was significantly higher than that at week 8 post-transplantation $(0.85 \pm 0.09$ vs. $0.76 \pm 0.23$, respectively, $p=0.03$ ). There was no difference in Shannon's diversity index during the postoperative period.

\section{Association between the plasma microbiome and clinical variations}

The post-transplant plasma microbiome and immunosuppressive medications were also investigated. There was no significant correlation between microorganism reads and tacrolimus concentration at the time of sample collection (Fig. 3). In addition, no significant correlation was detected between each microorganism and tacrolimus concentration (Additional file 2).

We obtained the microbiome of 75 samples after evaluating the quality of sequencing data using rarefaction curves. The non-metric multidimensional scaling (NMDS) plot of the microbiome of 75 samples showed that they tended to cluster together per time point (Fig. 4a). The analysis of reads per million mapped reads (RPM) profiles and clinical information (age, time point, tacrolimus, mycophenolate mofetil, steroids, antibacterial, antiviral, and antifungal) at the time of sample collection revealed that antimicrobials were associated with the microbiome. Samples collected after the administration of each antimicrobial tended to lean in the direction of each arrow (Fig. 4b-d). By contrast, age and immunosuppressive medications did not have significant correlations with the microbiome.

When patients were divided into two groups, children $(n=30)$ and adults $(n=81)$, there were some differences in individual microorganisms. At the family level, Enterobacteriaceae and Propionibacteriaceae were increased in the children group, whereas Anelloviridae, Nocardiaceae, and Methylobacteriaceae were increased in the adults group (Enterobacteriaceae: $0.24 \pm 0.16$ vs. $0.15 \pm 0.15$, respectively, $p=0.015$; Propionibacteriaceae: $0.11 \pm 0.10$ vs. $0.07 \pm 0.07$, respectively, $p=0.012$; Anelloviridae: $0.01 \pm 0.03$ vs. $0.11 \pm 0.22$, respectively, $p<0.001$; Nocardiaceae: $0.05 \pm 0.06$ vs. $0.09 \pm 0.10$, respectively,
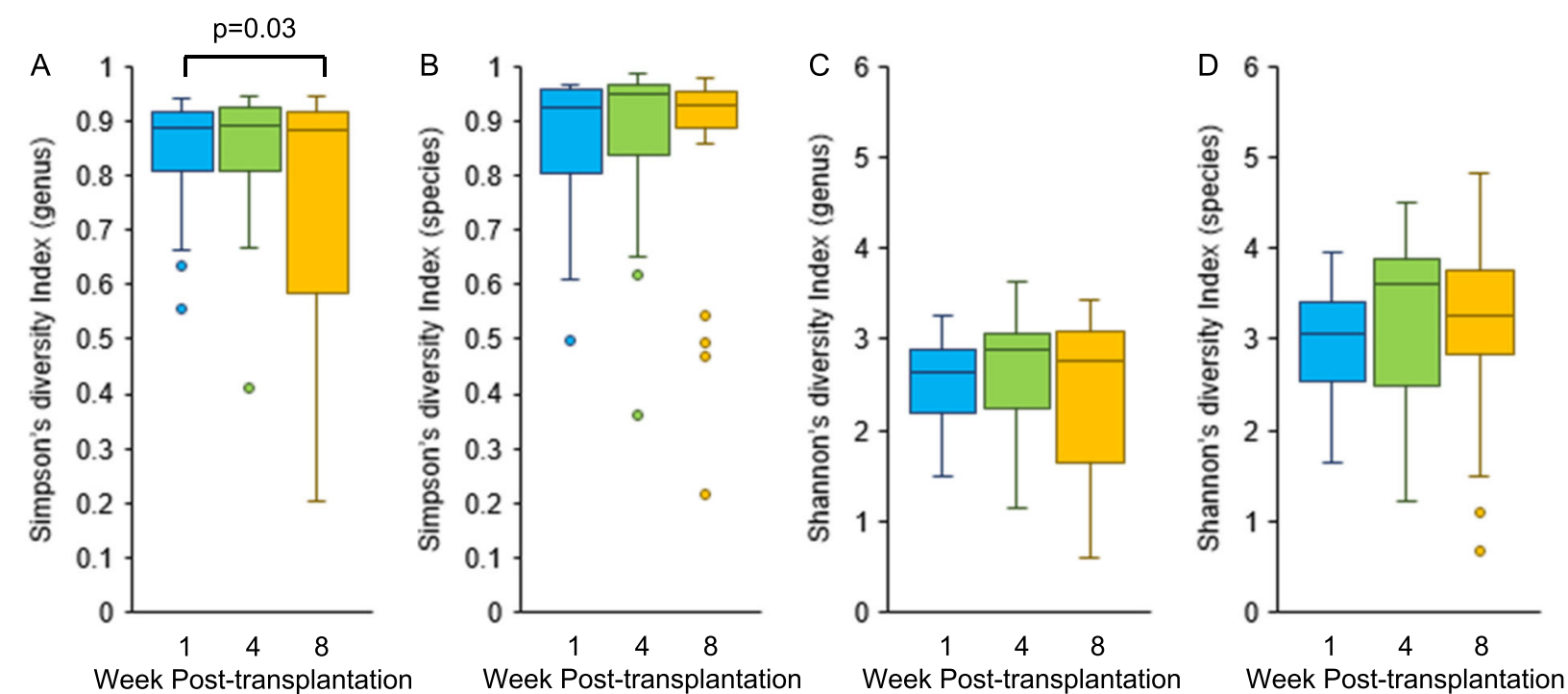

Fig. 2 Change in plasma microbiome diversity at genus and species levels after liver transplantation. a Simpson's diversity index at the genus level, b Simpson's diversity index at the species level, c Shannon's diversity index at the genus level, and d Shannon's diversity index at the species level at each sampling point are shown. Simpson's diversity index (at the genus level) of samples collected at week 1 post-transplantation was significantly higher than that of samples collected at week 8 post-transplantation ( $0.85 \pm 0.09$ vs. $0.76 \pm 0.23$, respectively, $p=0.03$ ) 


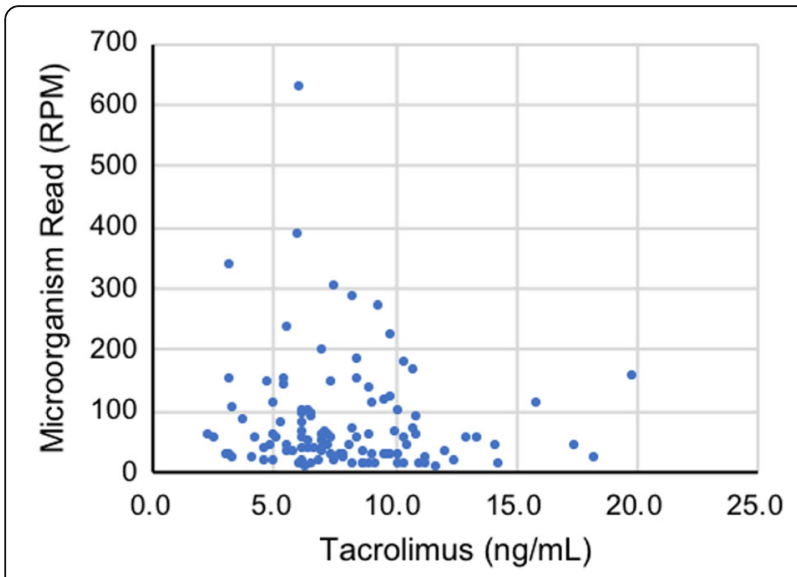

Fig. 3 Correlation between the number of microorganism reads and the tacrolimus concentration after liver transplantation. The number of microorganism reads and the tacrolimus concentration after liver transplantation are plotted. There was no significant correlation between the two indicators $(r=0.05, p=0.62)$. RPM, reads per million mapped reads $p=0.012$; Methylobacteriaceae: $0.01 \pm 0.02$ vs. $0.02 \pm 0.03$ respectively, $p=0.011$; Fig. S3).

\section{Association between the plasma microbiome and ACR}

Forty-six patients, whose samples were collected at week 1 post-transplantation, were included in the analysis to assess the association between the plasma microbiome and ACR. Table 1 shows the characteristics of patients in ACR and "no rejection" (NR) groups. Adult patients in the ACR group were significantly younger than those in the NR group $(p=0.02)$. The mean tacrolimus concentrations that had been examined before the development of ACR and in the first 2 weeks after LT in the NR group showed no significant difference. Comparing the average relative abundance at the family level, Xanthomonadaceae was increased in the ACR group, whereas Enterobacteriaceae was increased in the NR group (Xanthomonadaceae: $0.015 \pm 0.015$ vs. $0.005 \pm 0.007$, respectively, $p=0.047$; Enterobacteriaceae: $0.18 \pm 0.16$, vs. $0.30 \pm 0.17$, respectively, $p=0.045$; Fig. 5). Comamonadaceae and Nocardiaceae abundance tended to be higher in the ACR group than in the NR group, but there was no significant correlation. As shown in Fig. 6, Simpson's diversity indices in the ACR group were significantly higher than those in the NR group (genus level: $0.89 \pm$
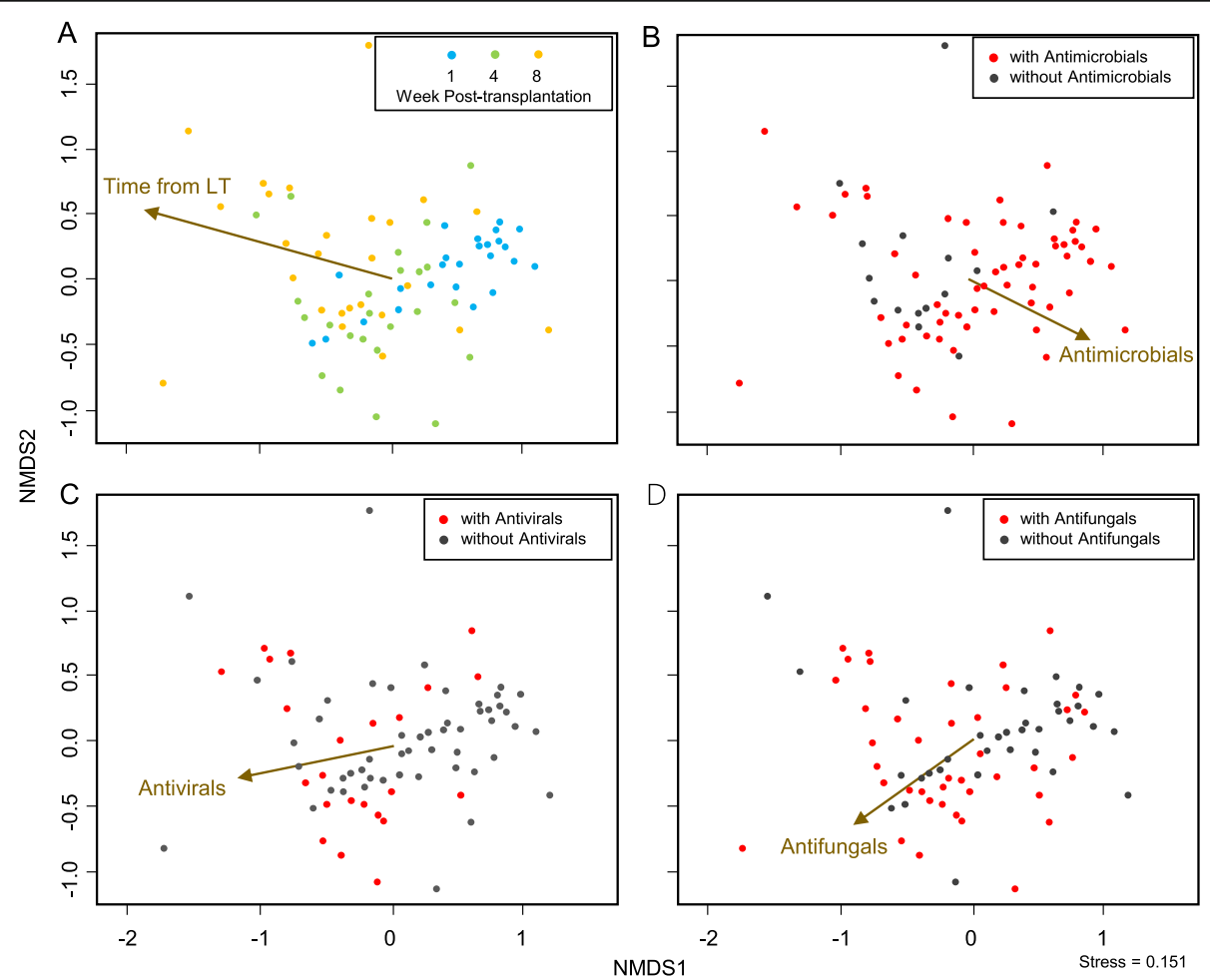

Fig. 4 Non-metric multidimensional scaling representation of plasma samples at each sampling point with envfit correlations. Samples were plotted based on Bray-Curtis distances calculated using family compositions (stress value $=0.151$ ). Arrows represent envfit correlations for a time from LT, $\mathbf{b}$ antimicrobials, $\mathbf{c}$ antivirals, and $\mathbf{d}$ antifungals; strong factors have longer arrows, whereas weak factors have shorter arrows. LT, liver transplantation; NMDS, non-metric multidimensional scaling 
Table 1 Patient characteristics and comparisons between acute cellular rejection (ACR) and no rejection (NR) groups

\begin{tabular}{|c|c|c|c|}
\hline & $\mathrm{ACR}(n=13)$ & NR $(n=33)$ & $p$-value \\
\hline No. patients (pediatric/adult) ${ }^{a}$ & $4 / 9$ & $8 / 25$ & 0.72 \\
\hline \multicolumn{4}{|l|}{ Age at LT [median (range)] } \\
\hline Pediatric & $13 \mathrm{~m}(5-50 \mathrm{~m})$ & $10.5 \mathrm{~m}(7-25 \mathrm{~m})$ & 0.93 \\
\hline Adult & 39 y $(18-63$ y) & 54 y $(24-66$ y) & 0.02 \\
\hline Sex (male/female) & $5 / 8$ & $11 / 22$ & 0.74 \\
\hline Living donor/brain death donor & $11 / 2$ & $25 / 8$ & 0.70 \\
\hline ABO incompatible & $3(23.1 \%)$ & $4(12.1 \%)$ & 0.39 \\
\hline Tacrolimus concentrations (mean \pm SD) & $7.7 \pm 1.5$ & $8.0 \pm 1.3$ & 0.59 \\
\hline $\mathrm{AST}(\text { mean } \pm \mathrm{SD})^{\mathrm{b}}$ & $197 \pm 155$ & $235 \pm 406$ & 0.75 \\
\hline $\mathrm{ALT}(\text { mean } \pm \mathrm{SD})^{\mathrm{b}}$ & $409 \pm 295$ & $318 \pm 366$ & 0.43 \\
\hline Initially treated with mycophenolate mofetil & $9(69.2 \%)$ & $20(60.6 \%)$ & 0.74 \\
\hline \multicolumn{4}{|l|}{ Underlying disease } \\
\hline Biliary atresia & $4(30.8 \%)$ & $6(18.2 \%)$ & 0.44 \\
\hline Primary sclerosing cholangitis & $2(15.4 \%)$ & $6(18.2 \%)$ & 1.00 \\
\hline Primary biliary cholangitis & $2(15.4 \%)$ & $3(9.1 \%)$ & 0.61 \\
\hline Fulminant hepatic failure & $1(7.7 \%)$ & $4(12.1 \%)$ & 1.00 \\
\hline Hepatitis B & $2(15.4 \%)$ & $2(6.1 \%)$ & 0.57 \\
\hline Hepatitis C & $1(7.7 \%)$ & $3(9.1 \%)$ & 1.00 \\
\hline Autoimmune hepatitis & $1(7.7 \%)$ & $2(6.1 \%)$ & 1.00 \\
\hline Others & $2(15.4 \%)$ & $7(21.2 \%)$ & \\
\hline Days of hospitalization after LT [median (range)] & $86(37-208)$ & $59(24-326)$ & 0.25 \\
\hline
\end{tabular}

0.04 vs. $0.84 \pm 0.10$, respectively, $p=0.03$; species level: $0.93 \pm 0.04$ vs. $0.85 \pm 0.13$, respectively, $p=0.004$ ).

\section{Comparison of NGS results in plasma samples with blood culture results}

Sixteen samples were collected within 2 days after positive blood culture. The same bacterial species isolated in blood culture were identified by NGS in 14 of 16 samples, and predominant pathogens in blood culture were identified in 8 samples (Nos. 16, 20, 54, 67, 101, 107, 108, and 109; Fig. 7). Of note, the microorganism that accounted for the highest relative abundance in sample No. 16 was human herpesvirus $6(\mathrm{HHV}-6)$ and that in sample No. 54 was Propionibacterium sp. oral taxon 193. Moreover, sample Nos. 107, 108, and 109 were obtained from the same patient who continuously suffered from bloodstream infection for over a month.

Given the days of interval between NGS sample collection and blood culture sample collection, predominant bacterial species were identical in 4 of 6 recipients when the two samples were collected on the same day. Conversely, predominant bacterial species were identical in 4 of 10 samples when the interval of sample collection was 1-2 days apart. Although we checked several samples collected a few days before blood culture collection, the same predominant bacteria were not identified by NGS.

An overwhelming amount of HHV-6 was detected in sample No. 111 (relative abundance $=99.9 \%$ ) with negative blood culture. The sample was collected from a 42year-old female who received LT for drug-induced acute liver failure. Due to vascular complications, she had a complicated postoperative course with rapid elevation of liver enzymes. Unfortunately, she died after a week despite intensive care.

\section{Discussion}

In this study, we examined the plasma microbiome of LT recipients using NGS. Majority of the bacteria are separated out by centrifugation during plasma prepping; however, several studies have examined the microbiome in plasma, which we believe is a well-established method $[13,14]$. In this study, 95.18-99.97\% of the available reads were of human origin, even from plasma samples, and deep sequencing by NGS enabled detection of the microorganism genome in the plasma samples. Most of the microorganisms that we detected may be their DNA constituents in the blood, except for bacteremia, because NGS can capture all genes in the samples if they are 


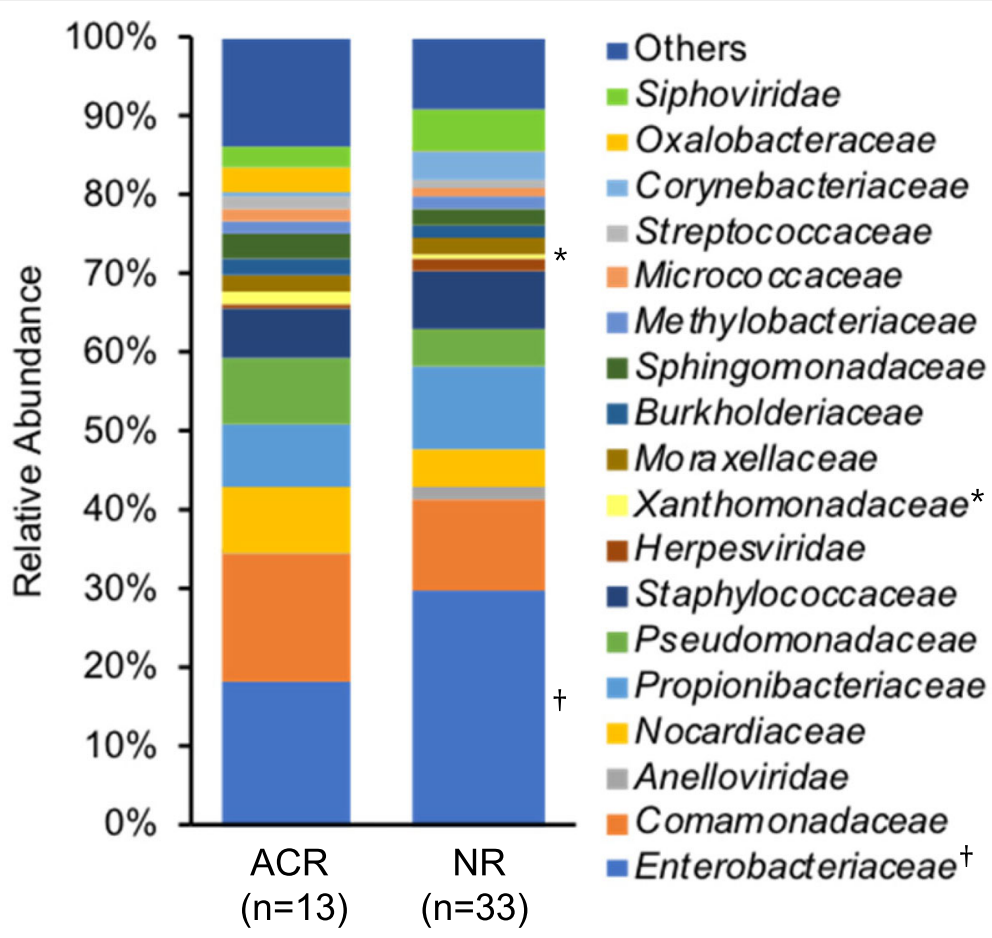

Fig. 5 Comparison of the plasma microbiome at the family level in patients with and without ACR. Xanthomonadaceae (*) abundance was significantly increased in the ACR group in comparison with the no rejection (NR) group $(p=0.047)$. In contrast, Enterobacteriaceae ( $(+)$ abundance was significantly decreased in the ACR group in comparison with the NR group $(p=0.045)$. ACR, acute cellular rejection; NR, no rejection
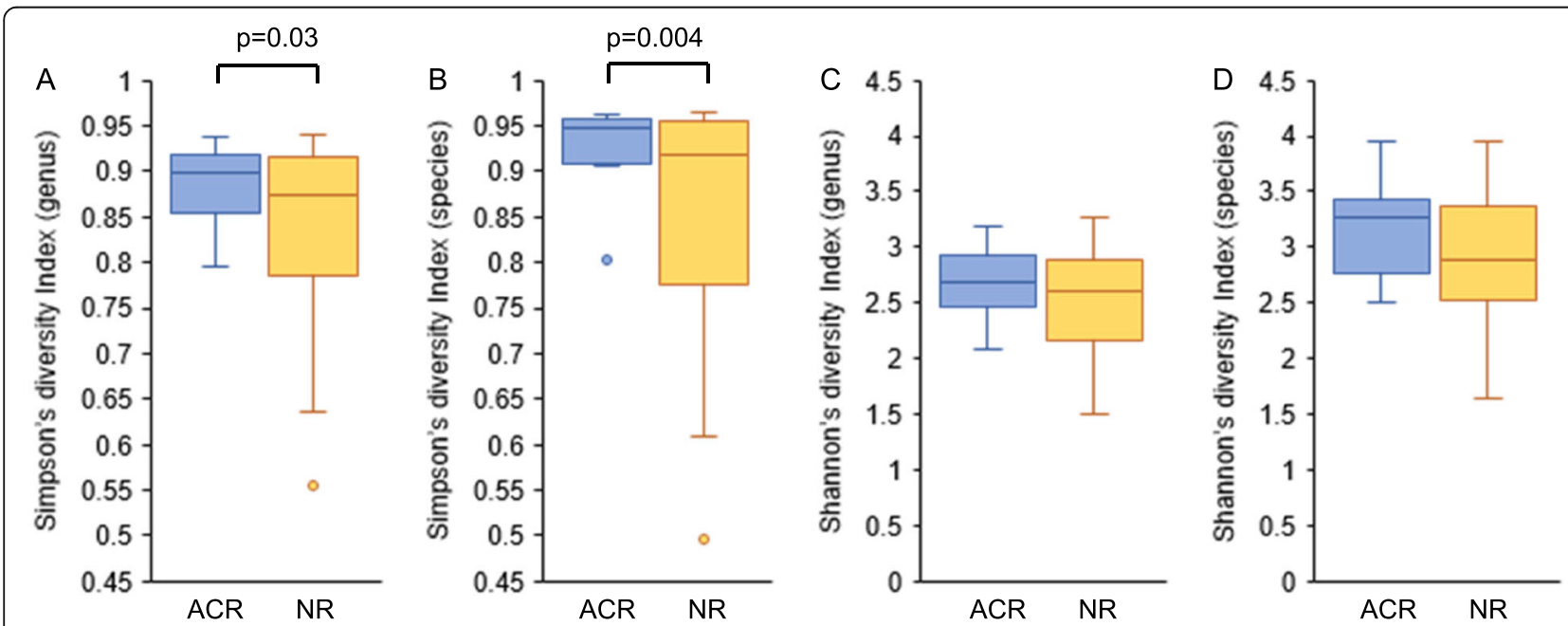

Fig. 6 Comparison of plasma microbiome diversity at genus and species levels in patients with and without ACR. a Simpson's diversity index at the genus level, b Simpson's diversity index at the species level, $\mathbf{c}$ Shannon's diversity index at the genus level, and d Shannon's diversity index at the species level are depicted. Simpson's diversity indices in the ACR group were significantly higher than those in the NR group (genus level: $0.89 \pm 0.04$ vs. $0.84 \pm 0.10$, respectively, $p=0.03$; species level: $0.93 \pm 0.04$ vs. $0.85 \pm 0.13$, respectively, $p=0.004)$. ACR, acute cellular rejection; NR, no rejection 


\begin{tabular}{|c|c|c|c|}
\hline No & Diagnosis & Bacteria Isolated in Blood Culture & Relative Abundance \\
\hline & CRBSI & Staphylococcus aureus & 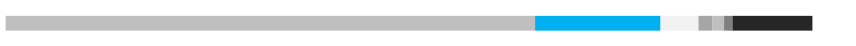 \\
\hline & FUO & Corynebacterium striatum & 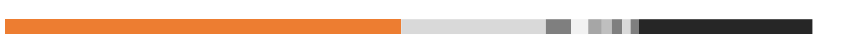 \\
\hline 54 & CRBSI & MRSA & | \\
\hline 62 & cholangitis & Escherichia coli & 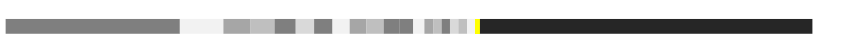 \\
\hline 67 & liver abscess & Enterococcus faecalis & Da \\
\hline 83 & CRBSI & Staphylococcus aureus & 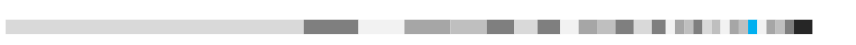 \\
\hline 97 & CRBSI & Enterobacter cloacae complex & be \\
\hline 99 & peritonitis & Acinetobacter baumannii & 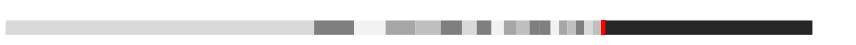 \\
\hline 101 & peritonitis & Stenotrophomonas maltophilia & 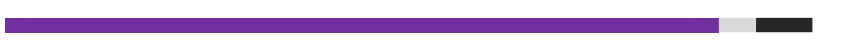 \\
\hline 105 & peritonitis & Enterococcus faecium & 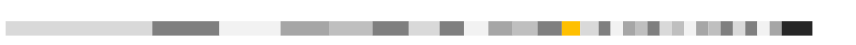 \\
\hline 106 & FUO & Haemophilus influenzae & 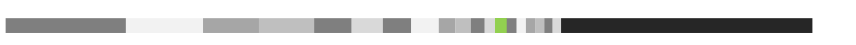 \\
\hline 107 & hepatic biloma & Enterococcus faecium & 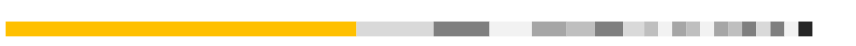 \\
\hline 108 & hepatic biloma & Enterococcus faecium & 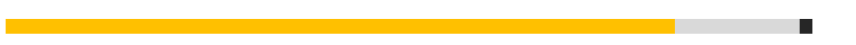 \\
\hline \multirow[t]{2}{*}{109} & hepatic biloma & Enterococcus faecium & 这 \\
\hline & & $0^{\circ}$ & $100 \%$ \\
\hline \multicolumn{4}{|c|}{$\begin{array}{l}\text { Fig. } 7 \text { Relative abundance of microorganisms at the species level in plasma from patients with positive blood cultures. Representative results of } \\
14 \text { patients are shown. Colored bars indicate that the same bacteria were isolated from next-generation sequencing and blood culture samples. } \\
\text { Black bars indicate microorganisms with }<1 \% \text { relative abundance. CRBSI, catheter-related bloodstream infection; FUO, fever of undetermined } \\
\text { origin; MRSA, methicillin-resistant Staphylococcus aureus }\end{array}$} \\
\hline
\end{tabular}

fragmented. The sources of these microorganisms are considered to be the gut or oral mucosa, from where they invade the blood $[15,16]$. Other sources may be contamination by normal skin-inhabiting microorganisms or even reagent contamination.

The plasma microbiome clearly changed from immediately after LT to 8 weeks post-transplantation. Interestingly, Anelloviridae and Enterobacteriaceae abundance dynamically changed during this period. The relative abundance of Anelloviridae increased and that of Enterobacteriaceae decreased in the postoperative course. The same trends were observed at the genus and species levels, but the amounts of microorganisms belonging to the genus and species were too small to identify individual microorganisms. Anelloviridae mainly consists of Torque teno virus (TTV). TTV, a small non-enveloped single-stranded DNA virus, is extremely common in the human population, and TTV DNA in plasma can be detected in about $75 \%$ of healthy individuals $[17,18]$. It is considered that TTV is not associated with any specific human illness at present [19]. Maggi et al. reported that TTV load in plasma from patients who received kidney or liver transplantation increased after transplantation [20]. Other studies also reported that TTV load in plasma or bronchoalveolar lavage from patients who received lung transplantation increased after transplantation $[21,22]$. In the present study, Anelloviridae was scarcely detected in samples immediately after LT and significantly increased several weeks later. This change indicated that Anelloviridae abundance increased under immunosuppressive therapy at a higher rate than other microorganisms. Although we expected that the number of microorganism reads would increase if immunosuppression therapy was strong, there was no significant correlation between the number of pathogen reads and tacrolimus concentration. In addition, there was no significant correlation between each microorganism and tacrolimus concentration in the present study. One possible reason is that tacrolimus concentration alone was insufficient to measure the degree of immunosuppression because immunosuppressive medication comprises not only tacrolimus but also mycophenolate mofetil and steroids. Comparing children and adults, they have different microbiomes, even in healthy individuals [23]. Furthermore, the underlying diseases were very different in the present study. In particular, children are rarely infected with Anelloviridae, which is rarely detected.

The NMDS plot of the microbiome showed high similarity of the microbiome among samples at the same time point. As antibiotics were administered prophylactically after operation, samples collected at week 1 posttransplantation would be classified as samples with antibiotics. Antifungals and antivirals were also associated with the microbiome. De Vlaminck et al. reported that dose of immunosuppressants and antivirals affect the viral component in plasma and the virome changes during the post-transplantation period. Herpesvirales and Caudovirales dominated the virome when patients received low doses of these drugs, and Anelloviridae dominated the virome when patients received high doses of these drugs [2]. Antibiotic treatment, infection, and LT itself were reported to be factors that change the structure of the bacterial component of the gut microbiome [24-27]. Our results showed that antimicrobial therapy was correlated with the plasma microbiome, but immunosuppressive medications were not. The change in the plasma microbiome may not be only due to the 
effect of antimicrobials, but also other factors under severe conditions in $\mathrm{LT}$ recipients.

In terms of ACR, patients in the NR group were expected to be more immunosuppressed in comparison with the ACR group because ACR indicates the development of strong immune responses. Comparison of the microbiome during the postoperative period indicated that samples collected at week 1 post-transplantation under strong immunosuppression had high relative abundance of $\mathrm{En}$ terobacteriaceae. The increase in relative abundance of Enterobacteriaceae may reflect the strength of immunosuppression. Previous studies reported that abundance of potentially pathogenic Enterobacteriaceae in gut microbiota increased in patients after LT $[1,28]$, and bacteria constituting the gut microbiota caused bloodstream infections [15]. Enterobacteriaceae detected in plasma was also considered to originate from the gut microbiota in the present study. No studies have hitherto referred to the link between ACR and leaky gut syndrome, but considering the results of this study, it is possible that Enterobacteriaceae easily invade the bloodstream from the gut in patients under strong immunosuppression. Moreover, Simpson's diversity indices in the NR group were lower than those in the ACR group. This result might be explained by the finding that Enterobacteriaceae accounted for nearly $30 \%$ of plasma microorganisms in the NR group. Enterobacteriaceae genome in plasma and the high diversity of the plasma microbiome may be a biomarker for the development of ACR after LT. Xanthomonadaceae abundance was increased in the ACR group, but its association with ACR was unclear because the amount of Xanthomonadaceae detected in this study was small. The causes and results of changes in the microbiome and the development of ACR are also unclear. If the microbiome is the cause, fecal microbiota transplantation or probiotics may prevent $\mathrm{ACR}$, but there are no reports of intervention at this time.

In this study, bacterial species identical to those isolated by blood culture were predominantly detected by NGS in 8 of 16 samples. The detection rate for bacterial species by NGS was comparable to rates reported by previous studies (50-70\%) [29-31]. However, detection rates of samples collected after sampling for blood cultures were rather low $(4$ of $10,40 \%)$ in this study. Bacteria were not detected by NGS in the samples collected before sampling for blood cultures, suggesting that the prediction of bloodstream infections by NGS is difficult. Previous studies [29-31] reported that pathogens could be detected by NGS in about $20 \%$ of culture-negative samples. Of note, an overwhelming abundance of HHV6 was detected in one patient when the patient's condition clinically worsened. NGS is expected to be a comprehensive diagnostic tool for pathogens in the clinical setting.
This study has several limitations. First, as this was a retrospective study, the details of blood sample collection were not available. Therefore, contamination cannot be excluded. Second, the recipients were heterogenous, particularly including adult and pediatric recipients. Third, there were no control samples before LT. Thus, the plasma microbiomes before and after LT were not compared. Whether the plasma microbiome after LT changes to the microbiome before LT is of great interest. Moreover, control samples from healthy individuals are necessary to know whether the microbiome of LT recipients becomes similar to that of healthy individuals.

\section{Conclusions}

In conclusion, the plasma microbiome and temporal dynamics in LT recipients were analyzed using NGS. Anelloviridae and Enterobacteriaceae abundance dynamically changed in the early phase of post-transplantation. The NMDS analysis revealed that antimicrobials are associated with the microbiome composition. Importantly, Enterobacteriaceae abundance and high plasma microbiome diversity may be indicators for the development of ACR after LT. NGS is useful as a comprehensive diagnostic tool for pathogens in LT recipients.

\section{Methods}

\section{Patients and samples}

Fifty-one patients who received LT at Nagoya University Hospital between 2016 and 2018 were enrolled in this study. Inclusion criteria were the availability of a plasma sample within a week after LT (presented as the sample collected at week 1 post-transplantation) or within 2 days after a positive blood culture. For some patients, we followed plasma samples $4 \pm 1$ weeks after LT (presented as the sample collected at week 4 post-transplantation) and $8 \pm 1$ weeks after LT (presented as the sample collected at week 8 post-transplantation). Table 2 summarizes the characteristics of the 51 patients (13 children and 38 adults). Underlying diseases for LT were biliary atresia in 10 patients followed by primary sclerosing cholangitis in 9 patients, primary biliary cholangitis in 6 patients, and fulminant hepatic failure in 6 patients.

ACR was defined as abnormal findings of liver tissue that indicated acute rejection through a biopsy performed on patients with clinical suspicion, such as fever and hepatic dysfunction, within 3 months after LT. We defined the ACR group as patients who developed ACR within 2 weeks after LT (13 of 19 patients with ACR) to evaluate the association between ACR and plasma microbiome at week 1 post-transplantation. The patient group without rejection was defined as patients with NR.

The median number of hospitalization days after LT was 62 days (range, 24-326 days). Immunosuppressive medications including tacrolimus and steroids were 
Table 2 Demographic characteristics of LT recipients

\begin{tabular}{|c|c|}
\hline & Total $(n=51)$ \\
\hline No. patients (pediatric/adult) ${ }^{a}$ & $13 / 38$ \\
\hline \multicolumn{2}{|l|}{ Age at LT [median (range)] } \\
\hline Pediatric & $12 \mathrm{~m}(5-50 \mathrm{~m})$ \\
\hline Adult & 50.5 y $(18-66$ y) \\
\hline Sex (male/female) & $19 / 32$ \\
\hline Living donor/brain death donor & $38 / 13$ \\
\hline ABO-incompatible & $8(15.7 \%)$ \\
\hline \multicolumn{2}{|l|}{ Underlying disease } \\
\hline Biliary atresia & $10(19.6 \%)$ \\
\hline Primary sclerosing cholangitis & $9(17.6 \%)$ \\
\hline Primary biliary cholangitis & $6(11.8 \%)$ \\
\hline Fulminant hepatic failure & $6(11.8 \%)$ \\
\hline Hepatitis B & $4(7.8 \%)$ \\
\hline Hepatitis C & $4(7.8 \%)$ \\
\hline Autoimmune hepatitis & $3(5.9 \%)$ \\
\hline Others & $11(21.6 \%)$ \\
\hline$A C R$ & $19(37.3 \%)$ \\
\hline Days from LT to ACR [median (range)] & $8(4-56)$ \\
\hline Days of hospitalization after LT [median (range)] & $62(24-326)$ \\
\hline
\end{tabular}

NOTE: Data are given as $\mathrm{n}(\%)$ unless otherwise noted. ${ }^{\mathrm{a}}$ One patient with hepatitis B developed fulminant hepatic failure, and another patient with hepatitis $B$ developed primary biliary cholangitis. $L T$ Liver transplantation, $A C R$ Acute cellular rejection

administered to all patients after LT. The target trough levels of tacrolimus were $10-15 \mathrm{ng} / \mathrm{mL}$ for the first 2 weeks, $5-10 \mathrm{ng} / \mathrm{mL}$ for the next 2 months, and $4-6 \mathrm{ng} /$ $\mathrm{mL}$ thereafter. Methylprednisolone was initially administered intravenously at a dose of $10 \mathrm{mg} / \mathrm{kg}$ immediately after reperfusion, followed by $1 \mathrm{mg} / \mathrm{kg}$ for the first 3 days, $0.5 \mathrm{mg} / \mathrm{kg}$ for the next 3 days, and $0.3 \mathrm{mg} / \mathrm{kg}$ on postoperative day 7 . Oral prednisolone was subsequently administered at a dose of $0.3 \mathrm{mg} / \mathrm{kg}$ once daily and tapered. Patients who received ABO blood-type incompatible transplants or had other indications for maintaining low tacrolimus concentrations, such as renal dysfunction, were treated with mycophenolate mofetil. Furthermore, adult patients who received $\mathrm{ABO}$ blood-type incompatible transplants were treated with rituximab and plasma exchange preoperatively and received additional methylprednisolone via the portal vein postoperatively. All patients received antibiotic prophylaxis with cefotaxime and ampicillin for $48 \mathrm{~h}$ postoperatively. Antifungal and antiviral prophylaxes were not routinely administered.

\section{Sample preparation and sequencing}

Collected plasma samples were stored at $-30^{\circ} \mathrm{C}$ until use. DNA was extracted from $140-\mu \mathrm{L}$ plasma samples using the QIAamp UCP Pathogen Mini Kit (Qiagen,
Hilden, Germany). DNA concentrations were measured using the Qubit dsDNA HS Assay Kit (Thermo Fisher Scientific, Waltham, MA, USA). DNA sequencing libraries were prepared using the Nextera XT Library Prep Kit (Illumina, San Diego, CA, USA) according to the manufacturer's instructions with slight modifications to increase the library concentration: in the clean up step, DNA libraries were purified twice using AMPure XP beads and final elution was carried out in $42.5 \mu \mathrm{L}$ of resuspension buffer. The library quality was analyzed using the Agilent 2100 bioanalyzer (Agilent, Santa Clara, CA, USA) and droplet digital PCR. DNA libraries were sequenced on the HiSeq system (Illumina) with the $2 \times$ $150 \mathrm{bp}$ paired-end protocol in the rapid run mode.

\section{NGS data processing}

To detect microbial sequences in plasma samples, NGS data files were imported into a newly developed bioinformatic pipeline named PATHDET. PATHDET is published on the internet and is free to use (PATHDET v1.0, https://pathdet.hgc.jp/). RPM of each microorganism were calculated in each plasma sample as the number of microorganism sequence reads per million sequence reads. Relative abundance was also calculated as the percentage of microorganism sequence reads in the total microorganism reads. Additionally, Simpson's diversity index and Shannon's diversity index based on each taxonomic hierarchy were calculated from the microorganism reads.

Next, we constructed a NMDS plot of microorganism beta-diversities measured by Bray-Curtis dissimilarity between RPM profiles at the family level using the $\mathrm{R}$ package vegan (https://CRAN.R-project.org/package = vegan). NMDS was used to visualize the level of similarity of the microbiome by considering dissimilarity distance matrices among samples. Before constructing the plot, we computed rarefaction curves to evaluate the quality of sequencing data. To identify factors that affect the microbiome, we compared RPM profiles with clinical information (age, time point, tacrolimus, mycophenolate mofetil, steroids, antibacterial, antiviral, and antifungal) at the time of sample collection using the function envfit. The envfit function fits environmental factors onto the ordination, with the length of the arrow proportional to the correlation with the ordination.

\section{Statistical analysis}

Continuous variables are presented as the median (range). Results were compared using Fisher's exact tests for categorical variables and the Mann-Whitney $U$ test for continuous variables. Index values, RPM, relative abundance, and each diversity index among the three time points were analyzed by analysis of variance and post hoc tests were conducted using the Bonferroni test. 
Correlations between RPM and tacrolimus concentration were performed using Pearson's correlation analysis and correlation coefficients $(r)$ were reported. Index values between ACR and NR groups were compared using Student's $t$-test. SPSS version 25.0 (IBM, Chicago, IL, USA) was used to perform the statistical analyses, and $p$-values $<0.05$, were considered statistically significant.

\section{Abbreviations}

ACR: Acute cellular rejection; CRBSI: Catheter-related bloodstream infection; FUO: Fever of undetermined origin; HHV-6: Human herpesvirus 6; LT: Liver transplantation; MRSA: Methicillin-resistant Staphylococcus aureus; NGS: Nextgeneration sequencing; NMDS: Non-metric multidimensional scaling; NR: No rejection; PCR: Polymerase chain reaction; RA: Relative abundance; RPM: Reads per million mapped reads; TTV: Torque teno virus

\section{Supplementary Information}

The online version contains supplementary material available at https://doi. org/10.1186/s12866-021-02154-W.

Additional file 1. The number of reads in each sample.

Additional file 2. Correlation between each microorganism and tacrolimus concentration.

Additional file 3: Fig. S1 Change in the relative abundance of each microorganism at the genus level in plasma samples after liver transplantation. Fig. S2 Change in the relative abundance of each microorganism at the species level in plasma samples after liver transplantation. Fig. $\mathbf{S 3}$ Comparison of the plasma microbiome at the family level in children and adults. Methylobacteriaceae $\left(^{*}\right)$, Nocardiaceae $(\ddagger)$, and Anelloviridae $(\S)$ abundances were significantly lower in children than in adults ( $p=0.011,0.012$, and $<0.001$, respectively). In contrast, the abundance of Propionibacteriaceae ( $\dagger$ ) and Enterobacteriaceae ( $\|)$ was significantly higher in children than in adults $(p=0.012$ and 0.015 , respectively).

\section{Acknowledgements}

Not applicable.

\section{Authors' contributions}

$\mathrm{TO1}, \mathrm{KH}$, and $\mathrm{YI}$ were responsible for all aspects of the study, including the study design, analysis and interpretation of results, and writing of the article. HK, ST, TS, YT1, JK, YT2, YO, and TO2 contributed the study design and revised the manuscript critically. All authors read and approved the final manuscript.

\section{Funding}

Not applicable.

\section{Availability of data and materials}

The datasets used and/or analyzed during the current study are available from the corresponding author on reasonable request.

\section{Declarations}

\section{Ethics approval and consent to participate}

The study was approved by the Institutional Review Board of Nagoya University Graduate School of Medicine (no. 9069). Written consent to participate was obtained from the parents/guardians of the minors included in this study (minors are considered anyone under the age of 16).

\section{Consent for publication}

Not applicable.

\section{Competing interests}

The authors declare that they have no competing interests.

\section{Author details}

'Department of Pediatrics, Nagoya University Graduate School of Medicine, 65 Tsurumai-cho, Showa-ku, Nagoya 466-8550, Japan. ${ }^{2}$ Department of Genetics, Research Institute of Environmental Medicine, Nagoya University, Nagoya, Japan. ${ }^{3}$ Department of Human Genetics and Molecular Biology, Nagoya University Graduate School of Medicine, Nagoya, Japan.

${ }^{4}$ Department of Transplantation Surgery, Nagoya University Hospital, Nagoya, Japan.

Received: 1 January 2021 Accepted: 16 March 2021

Published online: 06 April 2021

\section{References}

1. Doycheva I, Leise MD, Watt KD. The intestinal microbiome and the liver transplant recipient: what we know and what we need to know. Transplantation. 2016;100(1):61-8. https://doi.org/10.1097/TP.0000000000001 008.

2. De Vlaminck I, Khush KK, Strehl C, Kohli B, Luikart H, Neff NF, et al. Temporal response of the human virome to immunosuppression and antiviral therapy. Cell. 2013;155(5):1178-87. https://doi.org/10.1016/j.cell.2013.10.034.

3. Suzuki T, Kawada Jl, Okuno Y, Hayano S, Horiba K, Torii Y, Takahashi Y, Umetsu S, Sogo T, Inui A, Ito Y. Comprehensive detection of viruses in pediatric patients with acute liver failure using next-generation sequencing. J Clin Virol. 2017;96:67-72. https://doi.org/10.1016/j.jcv.2017.10.001.

4. Takeuchi S, Kawada Jl, Okuno Y, Horiba K, Suzuki T, Torii Y, Yasuda K, Numaguchi A, Kato T, Takahashi Y, Ito Y. Identification of potential pathogenic viruses in patients with acute myocarditis using next-generation sequencing. J Med Virol. 2018;90(12):1814-21. https://doi.org/10.1002/jmv.2 5263.

5. Mizrahi H, Peretz A, Lesnik R, Aizenberg-Gershtein Y, Rodríguez-Martínez S, Sharaby Y, Pastukh N, Brettar I, Höfle MG, Halpern M. Comparison of sputum microbiome of legionellosis-associated patients and other pneumonia patients: indications for polybacterial infections. Sci Rep. 2017;7(1):40114 https://doi.org/10.1038/srep40114.

6. Joensen KG, Engsbro AL $\varnothing$, Lukjancenko O, Kaas RS, Lund O, Westh $\mathrm{H}$, Aarestrup FM. Evaluating next-generation sequencing for direct clinical diagnostics in diarrhoeal disease. Eur J Clin Microbiol Infect Dis. 2017;36(7): 1325-38. https://doi.org/10.1007/s10096-017-2947-2.

7. Wang Z, Klipfell E, Bennett BJ, Koeth R, Levison BS, Dugar B, et al. Gut flora metabolism of phosphatidylcholine promotes cardiovascular disease. Nature. 2011:472(7341):57-63. https://doi.org/10.1038/nature09922.

8. Wang HF, Li LF, Guo SH, Zeng QY, Ning F, Liu WL, Zhang G. Evaluation of antibody level against Fusobacterium nucleatum in the serological diagnosis of colorectal cancer. Sci Rep. 2016;6(1):33440. https://doi.org/10.1 038/srep33440.

9. Qin N, Yang F, Li A, Prifti E, Chen Y, Shao L, Guo J, le Chatelier E, Yao J, Wu L, Zhou J, Ni S, Liu L, Pons N, Batto JM, Kennedy SP, Leonard P, Yuan C, Ding W, Chen Y, Hu X, Zheng B, Qian G, Xu W, Ehrlich SD, Zheng S, Li L. Alterations of the human gut microbiome in liver cirrhosis. Nature. 2014; 513(7516):59-64. https://doi.org/10.1038/nature13568.

10. Yoshimoto S, Loo TM, Atarashi K, Kanda H, Sato S, Oyadomari S, Iwakura Y, Oshima K, Morita H, Hattori M, Honda K, Ishikawa Y, Hara E, Ohtani N. Obesity-induced gut microbial metabolite promotes liver cancer through senescence secretome. Nature. 2013;499(7456):97-101. https://doi.org/10.1 038/nature12347.

11. Païssé S, Valle C, Servant F, Courtney M, Burcelin R, Amar J, Lelouvier B. Comprehensive description of blood microbiome from healthy donors assessed by 165 targeted metagenomic sequencing. Transfusion. 2016;56(5): 1138-47. https://doi.org/10.1111/trf.13477.

12. Moustafa A, Xie C, Kirkness E, Biggs W, Wong E, Turpaz Y, Bloom K, Delwart E, Nelson KE, Venter JC, Telenti A. The blood DNA virome in 8,000 humans. PLoS Pathog. 2017;13(3):e1006292. https://doi.org/10.1371/journal.ppat.1 006292.

13. Castillo DJ, Rifkin RF, Cowan DA, Potgieter M. The healthy human blood microbiome: fact or fiction? Front Cell Infect Microbiol. 2019;9:148. https:// doi.org/10.3389/fcimb.2019.00148.

14. Whittle E, Leonard MO, Harrison R, Gant TW, Tonge DP. Multi-method characterization of the human circulating microbiome. Front Microbiol. 2018;9:3266. https://doi.org/10.3389/fmicb.2018.03266.

15. Tamburini FB, Andermann TM, Tkachenko E, Senchyna F, Banaei N, Bhatt AS. Precision identification of diverse bloodstream pathogens in the gut 
microbiome. Nat Med. 2018;24(12):1809-14. https://doi.org/10.1038/s41591018-0202-8.

16. Lockhart PB, Brennan MT, Sasser HC, Fox PC, Paster BJ, Bahrani-Mougeot FK. Bacteremia associated with toothbrushing and dental extraction. Circulation 2008;117(24):3118-25. https://doi.org/10.1161/CIRCULATIONAHA.107.758524.

17. Haloschan M, Bettesch R, Görzer I, Weseslindtner L, Kundi M, PuchhammerStöckl E. TTV DNA plasma load and its association with age, gender, and HCMV IgG serostatus in healthy adults. Age (Dordr). 2014;36(5):9716. https:// doi.org/10.1007/s11357-014-9716-2.

18. Burra P, Masier A, Boldrin C, Calistri A, Andreoli E, Senzolo M, Zorzi M, Sgarabotto D, Guido M, Cillo U, Canova D, Bendinelli M, Pistello M, Maggi F, Palù G. Torque Teno virus: any pathological role in liver transplanted patients? Transpl Int. 2008;21(10):972-9. https://doi.org/10.1111/j.1432-2277.2 008.00714.x.

19. Focosi D, Antonelli G, Pistello M, Maggi F. Torquetenovirus: the human virome from bench to bedside. Clin Microbiol Infect. 2016;22(7):589-93. https://doi.org/10.1016/j.cmi.2016.04.007.

20. Maggi F, Focosi D, Statzu M, Bianco G, Costa C, Macera L, Spezia PG, Medici C, Albert E, Navarro D, Scagnolari C, Pistello M, Cavallo R, Antonelli G. Early post-transplant Torquetenovirus viremia predicts Cytomegalovirus reactivations in solid organ transplant recipients. Sci Rep. 2018;8(1):15490. https://doi.org/10.1038/s41598-018-33909-7.

21. Görzer I, Jaksch P, Kundi M, Seitz T, Klepetko W, Puchhammer-Stöckl E. Pretransplant plasma torque Teno virus load and increase dynamics after lung transplantation. PLoS One. 2015;10(3):e0122975. https://doi.org/10.1371/ journal.pone.0122975.

22. Abbas AA, Diamond JM, Chehoud C, Chang B, Kotzin JJ, Young JC, Imai I, Haas AR, Cantu E, Lederer DJ, Meyer KC, Milewski RK, Olthoff KM, Shaked A, Christie JD, Bushman FD, Collman RG. The perioperative lung transplant Virome: torque Teno viruses are elevated in donor lungs and show divergent dynamics in primary graft dysfunction. Am J Transplant. 2017; 17(5):1313-24. https://doi.org/10.1111/ajt.14076.

23. Derrien M, Alvarez AS, de Vos WM. The gut microbiota in the first decade of life. Trends Microbiol. 2019;27(12):997-1010. https://doi.org/10.1016/j.tim.201 9.08.001.

24. Jakobsson HE, Jernberg C, Andersson AF, Sjölund-Karlsson M, Jansson JK, Engstrand L. Short-term antibiotic treatment has differing long-term impacts on the human throat and gut microbiome. PLoS One. 2010;5(3): e9836. https://doi.org/10.1371/journal.pone.0009836.

25. Gollwitzer ES, Marsland BJ. Microbiota abnormalities in inflammatory airway diseases - potential for therapy. Pharmacol Ther. 2014;141(1):32-9. https:// doi.org/10.1016/j.pharmthera.2013.08.002.

26. Li L, Wang F, Liu Y, Gu F. Intestinal microbiota dysbiosis in children with recurrent respiratory tract infections. Microb Pathog. 2019;136:103709. https://doi.org/10.1016/j.micpath.2019.103709.

27. Bajaj JS, Fagan A, Sikaroodi M, White MB, Sterling RK, Gilles H, Heuman D, Stravitz RT, Matherly SC, Siddiqui MS, Puri P, Sanyal AJ, Luketic V, John B, Fuchs M, Ahluwalia V, Gillevet PM. Liver transplant modulates gut microbial dysbiosis and cognitive function in cirrhosis. Liver Transpl. 2017;23(7):90714. https://doi.org/10.1002/t.24754.

28. Wu ZW, Ling ZX, Lu HF, Zuo J, Sheng JF, Zheng SS, Li LJ. Changes of gut bacteria and immune parameters in liver transplant recipients. Hepatobiliary Pancreat Dis Int. 2012;11(1):40-50. https://doi.org/10.1016/s1499-3872(11 60124-0.

29. Miao Q, Ma Y, Wang Q, Pan J, Zhang Y, Jin W, et al. Microbiological diagnostic performance of metagenomic next-generation sequencing when applied to clinical practice. Clin Infect Dis. 2018;67(suppl_2):S231-S40. https://doi.org/10.1093/cid/ciy693.

30. Long Y, Zhang Y, Gong Y, Sun R, Su L, Lin X, Shen A, Zhou J, Caiji Z, Wang $X$, Li D, Wu H, Tan H. Diagnosis of Sepsis with cell-free DNA by nextgeneration sequencing technology in ICU patients. Arch Med Res. 2016; 47(5):365-71. https://doi.org/10.1016/j.arcmed.2016.08.004.

31. Horiba K, Kawada Jl, Okuno Y, Tetsuka N, Suzuki T, Ando S, Kamiya Y, Torii Y, Yagi T, Takahashi Y, Ito Y. Comprehensive detection of pathogens in immunocompromised children with bloodstream infections by nextgeneration sequencing. Sci Rep. 2018;8(1):3784. https://doi.org/10.1038/s41 598-018-22133-y.

\section{Publisher's Note}

Springer Nature remains neutral with regard to jurisdictional claims in published maps and institutional affiliations.

Ready to submit your research? Choose BMC and benefit from:

- fast, convenient online submission

- thorough peer review by experienced researchers in your field

- rapid publication on acceptance

- support for research data, including large and complex data types

- gold Open Access which fosters wider collaboration and increased citations

- maximum visibility for your research: over $100 \mathrm{M}$ website views per year

At BMC, research is always in progress.

Learn more biomedcentral.com/submissions 